J. Appl. Math. \& Informatics Vol. 32(2014), No. 3 - 4, pp. 377 - 387

http://dx.doi.org/10.14317/jami.2014.377

\title{
DIFFERENCE CORDIALITY OF SOME SNAKE GRAPHS
}

\author{
R. PONRAJ* AND S. SATHISH NARAYANAN
}

\begin{abstract}
Let $G$ be a $(p, q)$ graph. Let $f$ be a map from $V(G)$ to $\{1,2, \ldots, p\}$. For each edge $u v$, assign the label $|f(u)-f(v)| . f$ is called a difference cordial labeling if $f$ is a one to one map and $\left|e_{f}(0)-e_{f}(1)\right| \leq 1$ where $e_{f}(1)$ and $e_{f}(0)$ denote the number of edges labeled with 1 and not labeled with 1 respectively. A graph with admits a difference cordial labeling is called a difference cordial graph. In this paper, we investigate the difference cordial labeling behavior of triangular snake, Quadrilateral snake, double triangular snake, double quadrilateral snake and alternate snakes.
\end{abstract}

2010 AMS Mathematics Subject Classification : 05C78.

Key words and phrases : Triangular snake, Quadrilateral snake, Alternate, Alternate triangular snake, Alternate quadrilateral snake.

\section{Introduction}

Let $G$ be a $(p, q)$ graph. In this paper we have considered only simple and undirected graph. The number of vertices of $G$ is called the order of $G$, denoted by $|V(G)|$ and the number of edges of $G$ is called the size of $G$, denoted by $|E(G)|$. Labeled graphs are used in several areas such as astronomy, radar, circuit design and database management [1]. The notion of difference cordial labeling has been introduced by R. Ponraj, S. Sathish Narayanan and R. Kala in [3]. In $[3,4,5,6,7]$, difference cordial labeling behaviour of several graphs like path, cycle, complete graph, complete bipartite graph, bistar, wheel, web and some more standard graphs have been investigated. In this paper we investigate the difference cordial labeling behaviour of Triangular snake, Quadrilateral snake, Alternate triangular snake, Alternate quadrilateral snake. Let $x$ be any real number. Then $\lfloor x\rfloor$ stands for the largest integer less than or equal to $x$ and $\lceil x\rceil$ stands for the smallest integer greater than or equal to $x$. Terms and definitions not defined here are used in the sense of Harary [2].

Received May 13, 2013. Revised October 25, 2013. Accepted November 4, 2013.

* Corresponding author.

(C) 2014 Korean SIGCAM and KSCAM. 


\section{Main results}

Definition 2.1. Let $G$ be a $(p, q)$ graph. Let $f: V(G) \rightarrow\{1,2, \ldots, p\}$ be a bijection. Foreach edge $u v$, assign the label $|f(u)-f(v)|$. $f$ is called a difference cordial labeling if $f$ is $1-1$ and $\left|e_{f}(0)-e_{f}(1)\right| \leq 1$ where $e_{f}(1)$ and $e_{f}(0)$ denote the number of edges labeled with 1 and not labeled with 1 respectively. A graph with a difference cordial labeling is called a difference cordial graph.

Now we investigate the difference cordial labeling behavior of some snake graphs. The triangular snake $T_{n}$ is obtained from the path $P_{n}$ by replacing each edge of the path by a triangle $C_{3}$.

Theorem 2.2. The Triangular snake $T_{n}$ is difference cordial.

Proof. Let $P_{n}$ be the path $u_{1} u_{2} \ldots u_{n}$. Let $V\left(T_{n}\right)=V\left(P_{n}\right) \cup\left\{v_{i}: 1 \leq i \leq n-1\right\}$ and $E\left(T_{n}\right)=E\left(P_{n}\right) \cup\left\{u_{i} v_{i}, v_{i} u_{i+1}: 1 \leq i \leq n-1\right\}$. In this graph, $\left|V\left(T_{n}\right)\right|=$ $2 n-1$ and $\left|E\left(T_{n}\right)\right|=3 n-3$. For $n>4$, define $f: V\left(T_{n}\right) \rightarrow\{1,2, \ldots, 2 n-1\}$ by

$$
\begin{array}{rlrl}
f\left(u_{i}\right) & =2 i-1 & & \leq i \leq\left\lfloor\frac{n}{2}\right\rfloor \\
f\left(u_{\left\lfloor\frac{n}{2}\right\rfloor+i}\right) & =2\left\lfloor\frac{n}{2}\right\rfloor-1+i & 1 & \leq i \leq\left\lceil\frac{n}{2}\right\rceil \\
f\left(v_{n-i}\right) & =\left\lfloor\frac{n}{2}\right\rfloor+n-1+i & 1 & \leq i \leq\left\lceil\frac{n}{2}\right\rfloor \\
f\left(v_{i}\right) & =2 i & 1 & \leq i \leq\left\lfloor\frac{n}{2}\right\rfloor-1 .
\end{array}
$$

The following table 1 shows that the labeling $f$ defined above is a difference cordial labeing of $T_{n}$ for $n>4$.

TABLE 1

\begin{tabular}{|c|c|c|}
\hline Nature of $n$ & $e_{f}(0)$ & $e_{f}(1)$ \\
\hline$n \equiv 0(\bmod 2)$ & $\frac{3 n-4}{2}$ & $\frac{3 n-2}{2}$ \\
\hline$n \equiv 1(\bmod 2)$ & $\frac{3 n-3}{2}$ & $\frac{3 n-3}{2}$ \\
\hline
\end{tabular}

We now display a difference cordial labeling for $T_{2}, T_{3}$ and $T_{4}$ is given in figure 1.
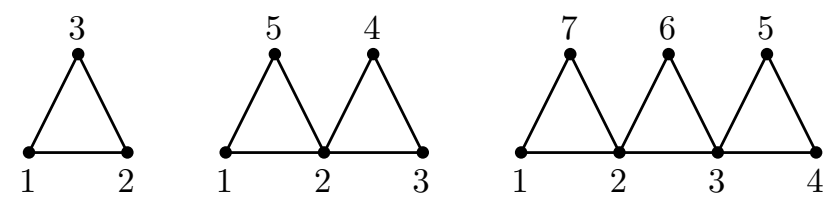

Figure 1.

The Quadrilateral snake $Q_{n}$ is obtained from the path $P_{n}$ by replacing each edge of the path by a cycle $C_{4}$. 
Theorem 2.3. All Quadrilateral snakes are difference cordial.

Proof. Let $P_{n}$ be the path $u_{1} u_{2} \ldots u_{n}$. Let $V\left(Q_{n}\right)=\left\{v_{i}, w_{i}: 1 \leq i \leq n-1\right\} \cup$ $V\left(P_{n}\right)$ and $E\left(Q_{n}\right)=E\left(P_{n}\right) \cup\left\{u_{i} v_{i}, v_{i} w_{i}, w_{i} u_{i+1}: 1 \leq i \leq n-1\right\}$. Note that $\left|V\left(Q_{n}\right)\right|=3 n-2$ and $\left|E\left(Q_{n}\right)\right|=4 n-4$. Define a map $f: V\left(Q_{n}\right) \rightarrow$ $\{1,2,3, \ldots, 3 n-2\}$ by $f\left(v_{1}\right)=3 n-3, f\left(v_{2}\right)=3 n-2, f\left(w_{1}\right)=3 n-4$,

$$
\begin{aligned}
f\left(u_{i}\right) & =i & & 1 \leq i \leq n \\
f\left(v_{n-i}\right) & =n+2 i & & 1 \leq i \leq n-3 \\
f\left(w_{n-i}\right) & =n+2 i-1 & & 1 \leq i \leq n-2 .
\end{aligned}
$$

Here, $e_{f}(0)=e_{f}(1)=2 n-2$. It follows that $f$ satisfies the edge condition of difference cordial graph.

Next is the alternate triangular snake. An alternate triangular snake $A\left(T_{n}\right)$ is obtained from a path $u_{1} u_{2} \ldots u_{n}$ by joining $u_{i}$ and $u_{i+1}$ (alternatively) to new vertex $v_{i}$. That is every alternate edge of a path is replaced by $C_{3}$.

Theorem 2.4. Alternate triangular snakes are difference cordial.

Proof. Case 1. Let the first triangle be starts from $u_{2}$ and the last triangle ends with $u_{n-1}$.

In this case, $\left|V\left(A\left(T_{n}\right)\right)\right|=\frac{3 n-2}{2}$ and $\left|E\left(A\left(T_{n}\right)\right)\right|=2 n-3$. Define $f$ : $V\left(A\left(T_{n}\right)\right) \rightarrow\left\{1,2, \ldots, \frac{3 n-2}{2}\right\}$ by $f\left(u_{1}\right)=1$,

$$
\begin{array}{rlrl}
f\left(u_{2 i}\right) & =3 i+1 & 1 & \leq i \leq \frac{n-2}{2} \\
f\left(u_{2 i+1}\right) & =3 i & 1 & \leq i \leq \frac{n-2}{2} \\
f\left(v_{i}\right) & =3 i-1 & 1 & \leq i \leq \frac{n-2}{2} .
\end{array}
$$

and $f\left(u_{n}\right)=\frac{3 n-2}{2}$. In this case $e_{f}(0)=n-1$ and $e_{f}(1)=n-2$ and hence $f$ is difference cordial labeling.

Case 2. Let the first triangle be starts from $u_{1}$ and the last triangle ends with $u_{n}$. Here $\left|V\left(A\left(T_{n}\right)\right)\right|=\frac{3 n}{2}$ and $\left|E\left(A\left(T_{n}\right)\right)\right|=2 n-1$. Define a map $f: V\left(A\left(T_{n}\right)\right) \rightarrow\left\{1,2, \ldots, \frac{3 n}{2}\right\}$ by

$$
\begin{array}{rlrl}
f\left(u_{2 i}\right) & =3 i-1 & & 1 \leq i \leq \frac{n}{2} \\
f\left(u_{2 i-1}\right) & =3 i-2 & & 1 \leq i \leq \frac{n}{2} \\
f\left(v_{i}\right) & =3 i & 1 & \leq i \leq \frac{n}{2} .
\end{array}
$$

Since $e_{f}(0)=n-1$ and $e_{f}(1)=n, f$ is a required difference cordial labeling. Case 3. Let the first triangle be starts from $u_{2}$ and the last triangle ends with $u_{n}$. Note that in this case, $\left|V\left(A\left(T_{n}\right)\right)\right|=\frac{3 n-1}{2}$ and $\left|E\left(A\left(T_{n}\right)\right)\right|=2 n-2$. Define 
an injective map $f: V\left(A\left(T_{n}\right)\right) \rightarrow\left\{1,2, \ldots, \frac{3 n-1}{2}\right\}$ by

$$
\begin{aligned}
& f\left(u_{1}\right)=\frac{3 n-1}{2} \\
& f\left(u_{4 i-2}\right)=6 i-5 \quad 1 \leq i \leq \frac{n-1}{4} \quad \text { if } \quad n \equiv 1(\bmod 4) \\
& 1 \leq i \leq \frac{n+1}{4} \quad \text { if } \quad n \equiv 3(\bmod 4) \\
& f\left(u_{4 i+1}\right)=6 i-2 \quad 1 \leq i \leq \frac{n-1}{4} \quad \text { if } \quad n \equiv 1(\bmod 4) \\
& 1 \leq i \leq \frac{n-3}{4} \quad \text { if } \quad n \equiv 3(\bmod 4) \\
& f\left(u_{4 i-1}\right)=3 i \quad 1 \leq i \leq \frac{n-1}{4} \quad \text { if } \quad n \equiv 1(\bmod 4) \\
& 1 \leq i \leq \frac{n+1}{4} \quad \text { if } \quad n \equiv 3(\bmod 4) \\
& f\left(u_{4 i}\right)=6 i \quad 1 \leq i \leq \frac{n-1}{4} \quad \text { if } \quad n \equiv 1(\bmod 4) \\
& 1 \leq i \leq \frac{n-3}{4} \quad \text { if } \quad n \equiv 3(\bmod 4) \\
& f\left(v_{i}\right)=3 i-1 \quad 1 \leq i \leq \frac{n-1}{2} .
\end{aligned}
$$

Here $e_{f}(0)=n-1$ and $e_{f}(1)=n-1$. Therefore, $f$ is a difference cordial labeling.

Case 4 . Let the first triangle be starts from $u_{1}$ and the last triangle ends with $u_{n-1}$. This case is equivalent to case 3 .

Now we look into alternate quadrilateral snake. An alternate quadrilateral snake $A\left(Q_{n}\right)$ is obtained from a path $u_{1} u_{2} \ldots u_{n}$ by joining $u_{i}, u_{i+1}$ (alternatively) to new vertices $v_{i}, w_{i}$ respectively and then joining $v_{i}$ and $w_{i}$. That is every alternate edge of a path is replaced by a cycle $C_{4}$.

Theorem 2.5. All alternate quadrilateral snakes are difference cordial.

Proof. Case 1. Let the first cycle $C_{4}$ be starts from $u_{2}$ and the last cycle be ends with $u_{n-1}$. Note that in this case, $\left|V\left(A\left(Q_{n}\right)\right)\right|=2 n-2$ and $\left|E\left(A\left(Q_{n}\right)\right)\right|=$ $\frac{5 n-8}{2}$. Define $f: V\left(A\left(Q_{n}\right)\right) \rightarrow\{1,2, \ldots, 2 n-2\}$ as follows:

$$
\begin{array}{rlrlrl}
f\left(u_{1}\right) & =1 & & & \\
f\left(v_{2 i-1}\right)=8 i-4 & 1 & \leq i \leq \frac{n}{4} & \text { if } & & n \equiv 0(\bmod 4) \\
& 1 \leq i \leq \frac{n-2}{4} & \text { if } & & n \equiv 2(\bmod 4) \\
f\left(v_{2 i}\right)=8 i+1 & 1 \leq i \leq \frac{n-4}{4} & \text { if } & & n \equiv 0(\bmod 4) \\
& 1 \leq i \leq \frac{n-2}{4} & \text { if } & & n \equiv 2(\bmod 4)
\end{array}
$$




$$
\begin{array}{rlrlrl}
f\left(w_{2 i-1}\right)=8 i-3 & 1 & \leq i \leq \frac{n}{4} & \text { if } & n \equiv 0(\bmod 4) \\
f\left(w_{2 i}\right)=6 i & 1 \leq i \leq \frac{n-2}{4} & \text { if } & n \equiv 2(\bmod 4) \\
& 1 \leq \frac{n-4}{4} & \text { if } & n \equiv 0(\bmod 4) \\
f\left(u_{2 i}\right)=4 i-2 & 1 \leq i \leq \frac{n-2}{2} & & & \\
f\left(u_{2 i+1}\right)=4 i-1 & 1 & & & \\
& 1 \leq i \leq \frac{n-2}{2} . & &
\end{array}
$$

The table 2 given below shows that $f$ is a difference cordial labeling.

TABLE 2

\begin{tabular}{|c|c|c|}
\hline Nature of $n$ & $e_{f}(0)$ & $e_{f}(1)$ \\
\hline$n \equiv 0(\bmod 4)$ & $\frac{5 n-8}{4}$ & $\frac{5 n-8}{4}$ \\
\hline$n \equiv 2(\bmod 4)$ & $\frac{5 n-10}{4}$ & $\frac{5 n-6}{4}$ \\
\hline
\end{tabular}

Case 2. Let the first cycle $C_{4}$ be starts from $u_{1}$ and the last cycle be ends with $u_{n}$. Here, $\left|V\left(A\left(Q_{n}\right)\right)\right|=2 n$ and $\left|E\left(A\left(Q_{n}\right)\right)\right|=\frac{5 n-2}{2}$. Define $f: V\left(A\left(Q_{n}\right)\right) \rightarrow$ $\{1,2, \ldots, 2 n\}$ by

$$
\begin{aligned}
& f\left(u_{4 i-3}\right)=8 i-7 \quad 1 \leq i \leq \frac{n}{4} \quad \text { if } \quad n \equiv 0(\bmod 4) \\
& 1 \leq i \leq \frac{n+2}{4} \quad \text { if } \quad n \equiv 2(\bmod 4) \\
& f\left(v_{4 i-2}\right)=8 i-6 \quad 1 \leq i \leq \frac{n}{4} \quad \text { if } \quad n \equiv 0(\bmod 4) \\
& 1 \leq i \leq \frac{n-2}{4} \quad \text { if } \quad n \equiv 2(\bmod 4) \\
& f\left(u_{4 i-1}\right)=8 i-3 \quad 1 \leq i \leq \frac{n}{4} \quad \text { if } \quad n \equiv 0(\bmod 4) \\
& 1 \leq i \leq \frac{n-2}{4} \quad \text { if } \quad n \equiv 2(\bmod 4) \\
& f\left(u_{4 i}\right)=8 i-2 \quad 1 \leq i \leq \frac{n}{4} \quad \text { if } \quad n \equiv 0(\bmod 4) \\
& 1 \leq i \leq \frac{n-2}{4} \quad \text { if } \quad n \equiv 2(\bmod 4) \\
& f\left(v_{2 i-1}\right)=8 i-5 \quad 1 \leq i \leq \frac{n}{4} \quad \text { if } \quad n \equiv 0(\bmod 4) \\
& 1 \leq i \leq \frac{n+2}{4} \quad \text { if } \quad n \equiv 2(\bmod 4) \\
& f\left(v_{2 i}\right)=8 i \quad 1 \leq i \leq \frac{n}{4} \quad \text { if } \quad n \equiv 0(\bmod 4)
\end{aligned}
$$




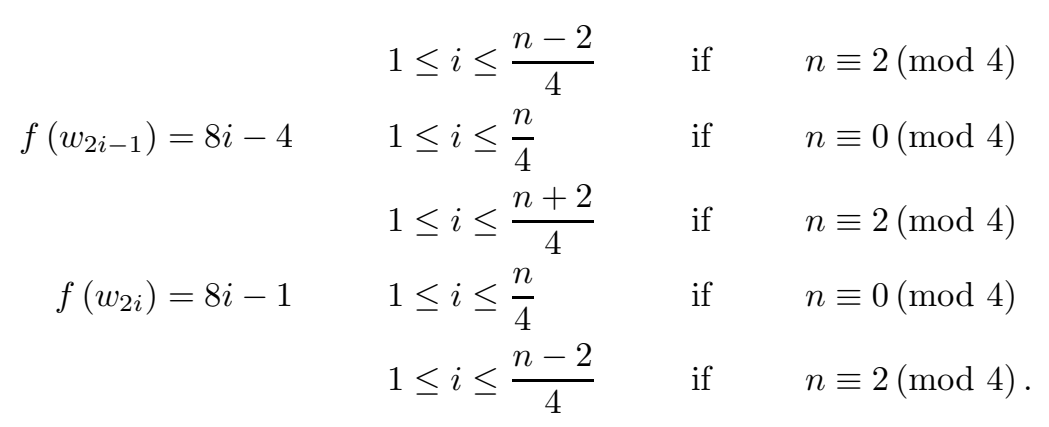

In this case the following table 3 shows that $f$ is a difference cordial labeling.

TABLE 3

\begin{tabular}{|c|c|c|}
\hline Nature of $n$ & $e_{f}(0)$ & $e_{f}(1)$ \\
\hline$n \equiv 0(\bmod 4)$ & $\frac{5 n-4}{4}$ & $\frac{5 n}{4}$ \\
\hline$n \equiv 2(\bmod 4)$ & $\frac{5 n-2}{4}$ & $\frac{5 n-2}{4}$ \\
\hline
\end{tabular}

Case 3. Let the first cycle $C_{4}$ be starts from $u_{2}$ and the last cycle be ends with $u_{n}$. Note that $\left|V\left(A\left(Q_{n}\right)\right)\right|=2 n-1$ and $\left|E\left(A\left(Q_{n}\right)\right)\right|=\frac{5 n-5}{2}$. Define $f: V\left(A\left(Q_{n}\right)\right) \rightarrow\{1,2, \ldots, 2 n-1\}$ by

$$
\begin{array}{rlrlrl}
f\left(u_{1}\right)=2 n-1 & & & \\
f\left(u_{4 i-2}\right)=8 i-7 & 1 \leq i \leq \frac{n-1}{4} & \text { if } & & n \equiv 1(\bmod 4) \\
f\left(u_{4 i-1}\right)=8 i-6 & 1 \leq i \leq \frac{n-1}{4} & \text { if } & & n \equiv 1(\bmod 4) \\
& 1 \leq i \leq \frac{n+1}{4} & \text { if } & & n \equiv 3(\bmod 4) \\
f\left(u_{4 i}\right)=8 i-3 & 1 \leq i \leq \frac{n-1}{4} & \text { if } & & n \equiv 1(\bmod 4) \\
f\left(u_{4 i+1}\right)=8 i-7 & 1 \leq i \leq \frac{n-1}{4} & \text { if } & & n \equiv 1(\bmod 4) \\
& 1 \leq i \leq \frac{n-3}{4} & \text { if } & & n \equiv 3(\bmod 4) \\
f\left(v_{2 i-1}\right)=8 i-4 & 1 \leq i \leq \frac{n-1}{4} & \text { if } & & n \equiv 1(\bmod 4) \\
& 1 \leq i \leq \frac{n+1}{4} & \text { if } & & n \equiv 3(\bmod 4) \\
f\left(v_{2 i}\right)=8 i-1 & 1 \leq i \leq \frac{n-1}{4} & \text { if } & & n \equiv 1(\bmod 4)
\end{array}
$$




$$
\begin{aligned}
& 1 \leq i \leq \frac{n-3}{4} \quad \text { if } \quad n \equiv 3(\bmod 4) \\
& f\left(w_{2 i-1}\right)=8 i-5 \quad 1 \leq i \leq \frac{n-1}{4} \quad \text { if } \quad n \equiv 1(\bmod 4) \\
& 1 \leq i \leq \frac{n+1}{4} \quad \text { if } \quad n \equiv 3(\bmod 4) \\
& f\left(w_{2 i}\right)=8 i \quad 1 \leq i \leq \frac{n-1}{4} \quad \text { if } \quad n \equiv 1(\bmod 4) \\
& 1 \leq i \leq \frac{n-3}{4} \quad \text { if } \quad n \equiv 3(\bmod 4) .
\end{aligned}
$$

The following table 4 shows that $f$ is a difference cordial labeling.

TABLE 4

\begin{tabular}{|c|c|c|}
\hline Nature of $n$ & $e_{f}(0)$ & $e_{f}(1)$ \\
\hline$n \equiv 1(\bmod 4)$ & $\frac{5 n-5}{4}$ & $\frac{5 n-5}{4}$ \\
\hline$n \equiv 3(\bmod 4)$ & $\frac{5 n-7}{4}$ & $\frac{5 n-3}{4}$ \\
\hline
\end{tabular}

Case 4. Let the first cycle $C_{4}$ be starts from $u_{1}$ and the last cycle be ends with $u_{n-1}$. This case is equivalent to case 3 .

Next investigation is about the irregular triangular snakes. The irregular triangular snake $I T_{n}$ is obtained from the path $P_{n}: u_{1} u_{2} \ldots u_{n}$ with vertex set $V\left(I T_{n}\right)=V\left(P_{n}\right) \rightarrow\left\{v_{i}: 1 \leq i \leq n-2\right\}$ and the edge set $E\left(I T_{n}\right)=E\left(P_{n}\right) \cup$ $\left\{u_{i} v_{i}, v_{i} u_{i+2}: 1 \leq i \leq n-2\right\}$.

Theorem 2.6. The irregular triangular snake is difference cordial.

Proof. Clearly $\left|V\left(I T_{n}\right)\right|=2 n-2$ and $\left|E\left(I T_{n}\right)\right|=3 n-5$. Define $f: V\left(I T_{n}\right) \rightarrow$ $\{1,2, \ldots, 2 n-2\}$ as follows:

Case 1. $n$ is odd.

$$
\begin{array}{rlrl}
f\left(u_{2 i-1}\right) & =2 i-1 & 1 & \leq i \leq\left\lceil\frac{n}{2}\right\rceil \\
f\left(u_{2 i}\right) & =2\left\lceil\frac{n}{2}\right\rceil+2 i-1 & 1 & \leq i \leq\left\lfloor\frac{n-2}{2}\right\rfloor \\
f\left(v_{2 i-1}\right) & =2 i & 1 & \leq i \leq\left\lceil\frac{n-2}{2}\right\rceil \\
f\left(v_{2 i}\right) & =2\left\lceil\frac{n-2}{2}\right\rceil+2 i & 1 & \leq i \leq\left\lfloor\frac{n-2}{2}\right\rfloor .
\end{array}
$$

Case 2. $n$ is even. Label the vertices $u_{i}(1 \leq i \leq n-1)$ and $v_{i}(1 \leq i \leq n)$ as in case 1 and assign the label $2 n-2$ to the vertex $u_{n}$. The following table 5 gives the nature of the edge condition of the above labeling $f$. It follows that $f$ is a difference cordial labeling.

The difference cordial labeling of irregular triangular snake $I T_{12}$ is given in figure 2 . 


\section{TABLE 5}

\begin{tabular}{|c|c|c|}
\hline Nature of $n$ & $e_{f}(0)$ & $e_{f}(1)$ \\
\hline$n \equiv 0(\bmod 2)$ & $\frac{3 n-4}{2}$ & $\frac{3 n-6}{2}$ \\
\hline$n \equiv 3(\bmod 2)$ & $\frac{3 n-5}{2}$ & $\frac{3 n-5}{2}$ \\
\hline
\end{tabular}

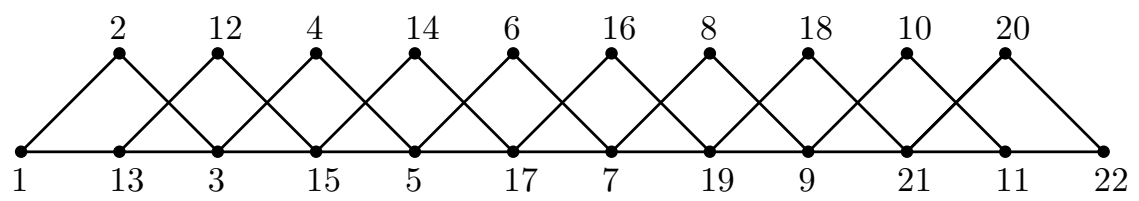

Figure 2 .

The irregular quadrilateral snake $I Q_{n}$ is obtained from the path $P_{n}: u_{1} u_{2} \ldots, u_{n}$ with vertex set $V\left(I Q_{n}\right)=V\left(P_{n}\right) \cup\left\{v_{i}, w_{i}: 1 \leq i \leq n-2\right\}$ and edge set $E\left(I Q_{n}\right)=$ $E\left(P_{n}\right) \cup\left\{u_{i} v_{i}, w_{i} u_{i+2}, v_{i} w_{i}: 1 \leq i \leq n-2\right\}$.

Theorem 2.7. The irregular quadrilateral snake is difference cordial.

Proof. Clearly, $\left|V\left(I Q_{n}\right)\right|=3 n-4$ and $\left|E\left(I Q_{n}\right)\right|=4 n-7$ respectively. Define $f: V\left(I Q_{n}\right) \rightarrow\{1,2,3, \ldots, 3 n-4\}$ by

$$
\begin{aligned}
f\left(u_{i}\right) & =i & & 1 \leq i \leq n \\
f\left(v_{i}\right) & =n+2 i-1 & & 1 \leq i \leq n-2 \\
f\left(w_{i}\right) & =n+2 i & & 1 \leq i \leq n-2 .
\end{aligned}
$$

Since $e_{f}(0)=\frac{4 n-8}{2}$ and $e_{f}(1)=\frac{4 n-6}{2}$, it follows that $f$ is a difference cordial labeling.

A double triangular snake $D T_{n}$ consists of two triangular snakes that have a common path. That is, a double triangular snake is obtained from a path $u_{1}, u_{2} \ldots u_{n}$ by joining $u_{i}$ and $u_{i+1}$ to a new vertex $v_{i}(1 \leq i \leq n-1)$ and to a new vertex $w_{i}(1 \leq i \leq n-1)$.

Theorem 2.8. Double triangular snake $D T_{n}$ is difference cordial iff $n \leq 6$.

Proof. For $n \leq 6$, the difference cordial labeling is given in figure 3. Conversely, suppose $n>6$ and $f$ is a difference cordial labeling of the double triangular snake. Here, $\left|V\left(D T_{n}\right)\right|=3 n-2$ and $\left|E\left(D T_{n}\right)\right|=5 n-5$. We observe that the maximum value of $e_{f}(1)$ does not exceed $1+2(n-1)+1=2 n$. Hence $e_{f}(0) \geq q-2 n \geq 3 n-5$. Therefore, $e_{f}(0)-e_{f}(1) \geq n-5$, a contradiction.

A double quadrilateral snake $D Q_{n}$ consists of two triangular snakes that have a common path. 

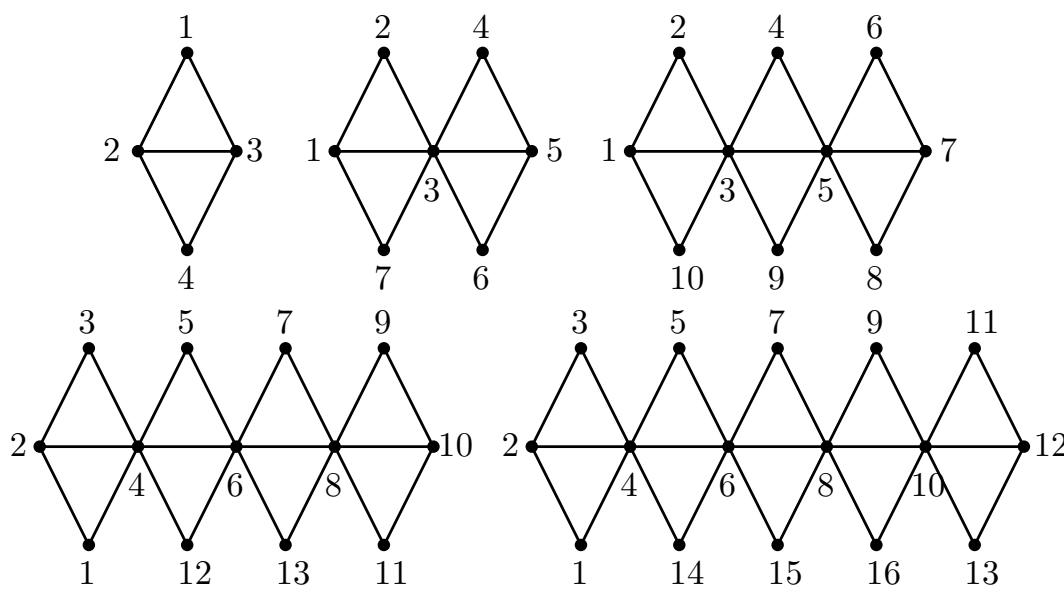

Figure 3 .

Theorem 2.9. The double quadrilateral snake is difference cordial.

Proof. Let $V\left(D Q_{n}\right)=\left\{u_{i}: 1 \leq i \leq n\right\} \cup\left\{v_{i}, w_{i}, x_{i}, y_{i}: 1 \leq i \leq n-1\right\}$ and $E\left(D Q_{n}\right)=\left\{u_{i} u_{i+1}, u_{i} v_{i}, v_{i} w_{i}, u_{i} x_{i}, x_{i} y_{i}, w_{i} u_{i+1}, y_{i} u_{i+1}: 1 \leq i \leq n-1\right\}$. Clearly, $\left|V\left(D Q_{n}\right)\right|=5 n-4$ and $\left|E\left(D Q_{n}\right)\right|=7 n-7$. Define a map $f: V\left(D Q_{n}\right) \rightarrow$ $\{1,2, \ldots, 5 n-4\}$ as follows:

$$
\begin{array}{rlrl}
f\left(u_{i}\right) & =3 i-2 & & 1 \leq i \leq\left\lfloor\frac{n+2}{2}\right\rfloor \\
f\left(v_{i}\right) & =3 i-1 & & 1 \leq i \leq\left\lfloor\frac{n}{2}\right\rfloor \\
f\left(w_{i}\right) & =3 i & & 1 \leq i \leq\left\lfloor\frac{n}{2}\right\rfloor \\
f\left(x_{i}\right) & =3 n+2 i-3 & & 1 \leq i \leq n-1 \\
f\left(y_{i}\right) & =3 n+2 i-2 & & 1 \leq i \leq n-1 \\
f\left(u_{\left\lfloor\frac{n+2}{2}\right\rfloor+i}\right) & =3\left\lfloor\frac{n+2}{2}\right\rfloor+i-2 & 1 & \left.1 \leq i \frac{n-2}{2}\right\rceil \\
f\left(v_{\left\lfloor\frac{n}{2}\right\rfloor+i}\right) & =3\left\lfloor\frac{n+2}{2}\right\rfloor+\left\lceil\frac{n-2}{2}\right\rceil+2 i-3 & & 1 \leq i \leq\left\lceil\frac{n}{2}\right\rceil-1 \\
f\left(w_{\left\lfloor\frac{n}{2}\right\rfloor+i}\right) & =3\left\lfloor\frac{n+2}{2}\right\rfloor+\left\lceil\frac{n-2}{2}\right\rceil+2 i-2 & & 1 \leq i \leq\left\lceil\frac{n}{2}\right\rceil-1 .
\end{array}
$$

The following table 6 shows that $f$ is a difference cordial labeling of $D Q_{n}$.

TABLE 6

\begin{tabular}{|c|c|c|}
\hline Nature of $n$ & $e_{f}(0)$ & $e_{f}(1)$ \\
\hline$n \equiv 0(\bmod 2)$ & $\frac{7 n-8}{2}$ & $\frac{7 n-6}{2}$ \\
\hline$n \equiv 1(\bmod 2)$ & $\frac{7 n-7}{2}$ & $\frac{7 n-7}{2}$ \\
\hline
\end{tabular}

A double alternate triangular snake $D A\left(T_{n}\right)$ consists of two alternate triangular snakes that have a common path. That is, a double alternate triangular snake is obtained from a path $u_{1} u_{2} \ldots u_{n}$ by joining $u_{i}$ and $u_{i+1}$ (alternatively) to two new vertices $v_{i}$ and $w_{i}$. 
Theorem 2.10. Double alternate triangular snake $D A\left(T_{n}\right)$ is difference cordial.

Proof. Case 1. The triangles starts from $u_{1}$ and end with $u_{n}$. In this case, $\left|V\left(D A\left(T_{n}\right)\right)\right|=2 n$ and $\left|E\left(D A\left(T_{n}\right)\right)\right|=3 n-1$. Define an injective map $f$ : $V\left(D A\left(T_{n}\right)\right) \rightarrow\{1,2, \ldots, 2 n\}$ by

$$
\begin{aligned}
& f\left(u_{i}\right)=2 i-1 \\
& 1 \leq i \leq n \\
& f\left(v_{i}\right)=4 i-2 \\
& 1 \leq i \leq \frac{n}{2} \\
& f\left(w_{i}\right)=4 i \\
& 1 \leq i \leq \frac{n}{2} .
\end{aligned}
$$

Since $e_{f}(1)=\frac{3 n}{2}$ and $e_{f}(0)=\frac{3 n-2}{2}, f$ is a difference cordial labeling of $D A\left(T_{n}\right)$. Case 2. The triangles starts from $u_{2}$ and end with $u_{n-1}$. Note that In this case, $\left|V\left(D A\left(T_{n}\right)\right)\right|=2 n-2$ and $\left|E\left(D A\left(T_{n}\right)\right)\right|=3 n-5$. Label the vertices $v_{i}$ and $w_{i}\left(1 \leq i \leq \frac{n-2}{2}\right)$ as in case 1 and define $f\left(u_{i}\right)=2 i-3,2 \leq i \leq n-1$, $f\left(u_{1}\right)=2 n-3$ and $f\left(u_{n}\right)=2 n-2$. Since $e_{f}(1)=\frac{3 n-6}{2}$ and $e_{f}(0)=\frac{3 n-4}{2}, f$ is a difference cordial labeling of $D A\left(T_{n}\right)$.

Case 3. The triangles starts from $u_{2}$ and end with $u_{n}$. It is clear that in this case, $\left|V\left(D A\left(T_{n}\right)\right)\right|=2 n-1$ and $\left|E\left(D A\left(T_{n}\right)\right)\right|=3 n-3$. Label the vertices $v_{i}$ and $w_{i}\left(1 \leq i \leq \frac{n-1}{2}\right)$ as in case 1 and label $u_{i}(2 \leq i \leq n-1)$ as in case 2 and define $f\left(u_{1}\right)=2 n-1$. Since $e_{f}(1)=e_{f}(0)=\frac{3 n-3}{2}, f$ is a difference cordial labeling of $D A\left(T_{n}\right)$.

Case 4. The triangles starts from $u_{1}$ and end with $u_{n-1}$. This case is equivalent to case 3 .

Finally, we look into the graph double alternate quadrilateral snake. A double alternate quadrilateral snake $D A\left(Q_{n}\right)$ consists of two alternate quadrilateral snakes that have a common path. That is, it is obtained from a path $u_{1} u_{2} \ldots u_{n}$ by joining $u_{i}$ and $u_{i+1}$ (alternatively) to new vertices $v_{i}, x_{i}$ and $w_{i}, y_{i}$ respectively and adding the edges $v_{i} w_{i}$ and $x_{i} y_{i}$.

Theorem 2.11. All double alternate quadrilateral snakes are difference cordial.

Proof. Case 1 The squares starts from $u_{1}$ and end with $u_{n}$. In this case, $\left|V\left(D A\left(Q_{n}\right)\right)\right|=3 n$ and $\left|E\left(D A\left(Q_{n}\right)\right)\right|=4 n-1$. Define a map $f: V\left(D A\left(Q_{n}\right)\right) \rightarrow$ $\{1,2, \ldots, 3 n\}$ by

$$
\begin{aligned}
& f\left(u_{i}\right)=3 i-1 \\
& f\left(v_{i}\right)=6 i-5 \\
& f\left(w_{i}\right)=6 i-2 \\
& f\left(x_{i}\right)=6 i-3 \\
& f\left(y_{i}\right)=6 i
\end{aligned}
$$

$$
\begin{aligned}
& 1 \leq i \leq n \\
& 1 \leq i \leq i \leq \frac{n}{2} \\
& 1 \leq i \leq i \leq \frac{n}{2} \\
& 1 \leq i \leq i \leq \frac{n}{2} \\
& 1 \leq i \leq i \leq \frac{n}{2} .
\end{aligned}
$$


Since $e_{f}(1)=2 n$ and $e_{f}(0)=2 n-1, f$ is a difference cordial labeling of $D A\left(Q_{n}\right)$.

Case 2. The squares starts from $u_{2}$ and end with $u_{n-1}$. In this case, $\left|V\left(D A\left(Q_{n}\right)\right)\right|$ $=3 n-4$ and $\left|E\left(D A\left(Q_{n}\right)\right)\right|=4 n-7$. Label the vertices $v_{i}, w_{i}, x_{i}, y_{i}\left(1 \leq i \leq \frac{n-2}{2}\right)$ as in case 1 and define $f\left(u_{i}\right)=3 i-4,2 \leq i \leq n$, and $f\left(u_{1}\right)=3 n-5$. Since $e_{f}(1)=2 n-3$ and $e_{f}(0)=2 n-4, f$ is a difference cordial labeling of $D A\left(Q_{n}\right)$. Case 3. The square starts from $u_{2}$ and end with $u_{n}$. In this case, $\left|V\left(D A\left(Q_{n}\right)\right)\right|=$ $3 n-2$ and $\left|E\left(D A\left(Q_{n}\right)\right)\right|=4 n-4$. Label the vertices $v_{i}, w_{i}, x_{i}, y_{i}\left(1 \leq i \leq \frac{n-1}{2}\right)$ as in case 1 and label $u_{i}(2 \leq i \leq n)$ as in case 2 and define $f\left(u_{1}\right)=3 n-2$. Since $e_{f}(1)=e_{f}(0)=2 n-2, f$ is a difference cordial labeling of $D A\left(Q_{n}\right)$.

Case 4. The square starts from $u_{1}$ and end with $u_{n-1}$. This case is equivalent to case 3 .

Acknowledgement. The authors thank the refree for his suggestions and valuable comments.

\section{REFERENCES}

1. J. A. Gallian, A Dynamic survey of graph labeling, The Electronic Journal of Combinatorics, 19 (2012) \# Ds6.

2. F. Harary, Graph theory, Addision wesley, New Delhi (1969).

3. R. Ponraj, S. Sathish Narayanan and R. Kala, Difference cordial labeling of graphs, Global Journal of Mathematical Sicences: Theory and Practical, 5(2013), 185-196.

4. R.Ponraj, S.Sathish Narayanan, R.Kala, Difference cordial labeling of graphs obtained from Double snakes, International Journal of Mathematics Research, 5(2013), 317-322.

5. R.Ponraj, S.Sathish Narayanan, Difference cordiality of some graphs obtained from Double Alternate Snake Graphs, Global Journal of Mathematical Sciences: Theory and Practical, 5(2013), 167-175.

6. R. Ponraj, S. Sathish Narayanan and R. Kala, Difference cordial labeling of corona graphs, $J$. Math. Comput. Sci., 3(2013), 1237-1251.

7. R. Ponraj and S. Sathish Narayanan, Further results on difference cordial labeling of corona graphs, The Journal of the Indian Academy of Mathematics, 35(2013).

Dr. R. Ponraj received his Ph.D in Manonmaniam Sundaranar University, Tirunelveli. He is currently an Assistant Professor at Sri Paramakalyani College, Alwarkurichi, India. His Research interest is in Discrete Mathematics.

Department of Mathematics, Sri Paramakalyani College, Alwarkurichi, Tamil Nadu, India627412 .

e-mail: ponrajmaths@gmail.com

Mr. S. Sathish Narayanan did his M.Phil in St. John's College, Palayamkottai. He is persuing doctoral research work. His Research interest is in Graph labeling.

Department of Mathematics, Sri Paramakalyani College, Alwarkurichi, Tamil Nadu 627 412, India.

e-mail: sathishrvss@gmail.com 